\title{
COMPARISON OF THREE MODELS OF AVALANCHE DYNAMICS
}

\author{
by \\ H. Gubler \\ (Eidgenössisches Institut für Schnee- und Lawinenforschung, Weissfluhjoch, \\ CH-7260 Davos, Switzerland)
}

\section{ABSTRACT}

Results and characteristics of three models for estimating avalanche flow speeds, flow heights, and run-out distances are compared: (1) Voellmy-Salm equation used with the traditional release, track, and run-out segmentation method; (2) Voellmy-Salm differential equation solved numerically along longitudinal profiles of avalanche paths, combined with modified assumptions for the flow in the run-out zone; (3) a granular-flow model introduced by Salm and Gubler. Within the limits of the accuracy of the field observations, all models are able to predict run-out distances correctly, at least for large avalanches, but the Voellmy-Salm type models significantly underestimate flow speeds. Modelling different flow regimes (sliding and partial fluidization) increases the range of avalanche sizes for which correct run-out modelling is possible without recalibration of model parameters.

\section{LIST OF PARAMETERS}

\section{General parameters}

$\begin{array}{ll}g & \left(\mathrm{~m} / \mathrm{s}^{2}\right) \\ x & (\mathrm{~m}) \\ s & (\mathrm{~m}) \\ x_{1}: x_{2} & \\ y & (\mathrm{~m}) \\ t & (\mathrm{~s}) \\ u_{0}(x) & (\mathrm{m} / \mathrm{s})\end{array}$

\section{$u(x, y) \quad(\mathrm{m} / \mathrm{s})$}

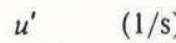

$d(x) \quad(\mathrm{m})$

$d_{\mathrm{E}} \quad(\mathrm{m})$

$\begin{array}{ll}d_{\mathrm{F}} & (\mathrm{m}) \\ d_{\mathrm{V}} & (\mathrm{m})\end{array}$

$A_{\mathrm{R}} \quad(\mathrm{m})$

$\lambda_{\mathrm{R}} \quad(\mathrm{m})$

$R_{\mathrm{R}}$

$Q(x) \quad\left(\mathrm{m}^{3} / \mathrm{s}\right)$

$V(x) \quad\left(\mathrm{m}^{3}\right)$

$m(x) \quad(\mathrm{kg})$

$L(x) \quad(\mathrm{m})$

$B(x) \quad(\mathrm{m})$

$F(x) \quad\left(\mathrm{m}^{2}\right)$

$U(x) \quad(\mathrm{m})$

$r(x) \quad(\mathrm{m})$

$T(x) \quad\left(\mathrm{m}^{2} / \mathrm{s}^{2}\right)$

$\rho \quad\left(\mathrm{kg} / \mathrm{m}^{3}\right)$

$\rho_{\mathrm{s}} \quad\left(\mathrm{kg} / \mathrm{m}^{3}\right)$

$\psi(x) \quad\left({ }^{\circ}\right)$

$l(x) \quad(\mathrm{m})$

$k_{\mathrm{i}} \quad\left(\mathrm{m} / \mathrm{s}^{2}\right)$

$\vec{v}_{0} \quad(\mathrm{~m} / \mathrm{s})$ acceleration due to gravity

coordinate along avalanche path

deposition front

from $x_{1}$ to $x_{2}$

coordinate perpendicular to slope

time

characteristic avalanche speed: flow speed (VS and VSG models), flow speed parallel to slope at surface of dense flow

time-averaged particle speed parallel to slope (speed fluctuations disregarded)

$u^{\prime}=\partial u / \partial x$

flow height

height of entrainable snow layer height of fluidized layer

height of layer leaving the flow

roughness amplitude

roughness wavelength

roughness parameter $\left(=A_{\mathrm{R}} / \lambda_{\mathrm{R}}\right)$

flow rate

avalanche volume

mass

length of avalanche body

flow or avalanche width

cross-sectional area of avalanche

wetted circumference of flow

hydraulic radius of flow $(=F / U)$

fluctuation energy per unit mass

density of avalanche snow

density of entrainable snow in track slope angle

relaxation distance to normal flow contribution to retardation of flow mean fluctuation speed of finegrained material in basal shear layer (scalar) $\bar{w}_{0} \quad(\mathrm{~m} / \mathrm{s})$

Indices

$r$
$t$
$o$
$s$
$e$
$p$

release zone

track

run-out

avalanche front

estimate

at point $\mathrm{P}$

\section{Model parameters}

$\begin{array}{ll}\mu & \\ \xi & \left(\mathrm{m} / \mathrm{s}^{2}\right) \\ \lambda_{\mathrm{V}} & \\ c_{\mathrm{S}} & \\ \lambda & (\mathrm{m}) \\ e & \\ \theta_{\mathrm{F}} & (\mathrm{m}) \\ \theta^{*} & (\mathrm{~m}) \\ \sigma^{*} & \left(\mathrm{~J} / \mathrm{m}^{2}\right)\end{array}$

dry-friction coefficient

friction coefficient for Voellmy type models

internal friction coefficient

shear-friction coefficient for PF model

decay length of coarse-grain fluctuation

coefficient of restitution

mean fine-grain radius

mean coarse-grain radius

effective surface energy of snow

\section{INTRODUCTION}

The Voellmy-Salm (VS) model used with the traditional release, track, and run-out segmentation method has for many years been used in consulting work to estimate extreme run-out distances. To ease some of the problems introduced with the segmentation of an avalanche path, the VS differential equations are integrated numerically along longitudinal profiles of avalanche paths (VSG model). To test different theoretical and qualitative assumptions, an experimental granular flow model, the partly fluidized flow (PF) model (Salm and Gubler, 1985), has been introduced. A set of 25 extreme avalanche occurrences, with estimated return periods in the range 30-300 years and showing extreme run-out, has been used to calibrate the VS and VSG model parameters for correct run-out distance matching. The PF model has been calibrated from speed and run-out data from flow-velocity measurements made using radar (Gubler, 1987).

\section{MODELLING}

A common problem to all models is that of the estimation of characteristic slab thickness and area in an avalanche-release zone; only in very few cases are reliable measurements available. In most cases the release area must be estimated from topographical restrictions and possible wind action. Statistical values dependent on mean return period and slope angle have been used to obtain mean fracture heights. These are evaluated from extreme-value statistics of new snow depth by Salm and Burkard (1988). 
In estimating slab volume, it is of ten necessary to make use of such statistics, which may require correction for snow redistribution by wind and for partial release of old snow. For the VSG and PF models, longitudinal profiles are digitized from topographical maps, whereas for the VS model segment lengths and mean slope angles for the release zone, the run-out approach, and the run-out are directly determined from such maps. In addition, restrictions of avalanche-flow width and track cross-section profiles have to be established from maps. This preparatory work can be done very quickly using interactive computer codes.

\section{VS model}

The VS model (Salm, 1984) includes continuity, assuming steady flow in the track and position-independent, non-steady sliding in the run-out. The model has been calibrated against observed run-out distances many times in the past (Buser and Frutiger, 1979). Gubler (1987) has attempted to calibrate the model against measured avalanche speeds and run-out distances, with results showing a clear dependence of the resulting friction parameters, $\mu, \xi$, on flow rate and avalanche size. Higher flow rates or flow heights are found to require lower friction and therefore produce higher speeds. To be able to fit speeds and run-out to the model, it was necessary to split the dry-friction coefficient into low track (high flow speed) and high run-out (low flow speed) values. Schaerer (1975) has proposed a similar speed dependence for $\mu$. A fast-stop mechanism seems to be characteristic of many avalanches; the main equations for the VS model are given below (see also parameter list at end of paper).

Flow speed and flow rate at the stauchwall

$$
\begin{gathered}
u_{\mathrm{or}}=\left[\mathrm{d}_{\mathrm{r}} \xi\left(\sin \psi_{\mathrm{r}}-\mu \cos \psi_{\mathrm{r}}\right)\right]^{\frac{1}{2}} \\
Q_{\mathrm{r}}=B_{\mathrm{r}} d_{\mathrm{r}} u_{\mathrm{or}} .
\end{gathered}
$$

Speed at point $\mathrm{P}$, the beginning of the run-out,

$$
u_{\mathrm{op}}=\left[r \xi\left(\sin \psi_{\mathrm{t}}-\mu \cos \psi_{\mathrm{t}}\right)\right]^{\frac{1}{2}}
$$

where

$$
r=F / U=\frac{B_{\mathrm{t}} d_{\mathrm{p}}}{B_{\mathrm{t}}+2 d_{\mathrm{p}}}
$$

for rectangular cross-section and

$$
Q_{\mathrm{p}}=F u_{\mathrm{op}}=Q_{\mathrm{r}} \text { (continuity). }
$$

Relaxation distance to which the track segment length $l_{p}$ must be matched for an arbitrary cross-section

$$
l_{\mathrm{p}}=0.7 \xi d_{\mathrm{p}} / g \text { with } d_{\mathrm{p}}=\frac{u_{o p}^{2}}{\xi\left(\sin \Psi_{\mathrm{t}}-\mu \cos \Psi_{\mathrm{t}}\right)} .
$$

Mean deposition height and run-out distance are represented by

$$
\begin{gathered}
d_{0}=\frac{d_{\mathrm{p}}+u_{\mathrm{op}}^{2}}{4 g \lambda_{\mathrm{v}}} \\
s_{0}=\left(d_{0} \xi / 2 g\right) \ln \left\{\lambda+\frac{u_{\mathrm{op}}^{2}}{d_{0} \xi\left(\mu \cos \psi_{0}-\sin \psi_{0}\right)}\right\} .
\end{gathered}
$$

One of the most critical decisions is the determination of the end-point of the characteristic track segment for the run-out approach. Basically, the steady-flow assumption that $Q=$ constant is replaced by the non-steady slide assumption at the point $\mathrm{P}$ where the local slope angle is equal to $\tan ^{-1} \mu$. If, above $P$, the slope angle increases only slightly, then the end-point of the run-out approach segment must be shifted further up-hill until the slope angle exceeds $\tan ^{-1} \mu$ by $3.5^{\circ}$ (Salm and Burkard, 1988). The values for flow speed and flow height calculated for this segment must be used as starting values at point $P$ for the run-out determination. In the event of changes in the characteristics of the slope angle in the run-out zone further segmentation is indicated. Starting speeds for consecutive segments are determined by linear interpolation of the square of the sliding speed. The VS model tends to predict very large deposition heights. This follows from the rather extreme assumption that the higher momentum of the rear section of the flow is transformed into increased flow height without pushing on the front section. The VSG model has been employed in order to relax some of the above-mentioned assumptions and rules.

\section{VSG model}

The VSG model differs from the VS model in two main respects. There are no straight-line approximations of the longitudinal profile. The beginning of the run-out (switching from flowing to sliding) is basically determined by the code from the digitized profile by searching for the point or points where $\tan \Psi=\mu_{0}$; as an additional option the sliding motion in the run-out can be modelled as a flexible but solid slab of varing width and thickness. Internal stresses in the avalanche body are taken into account by replacing the local slope angle for the front element at position, $x$, by a mean slope angle taken between $x$ and $x-l(x)$ for the steady-state flow regime, or between $x$ and $x_{\mathrm{p}}$ respectively over the actual length of the avalanche for the sliding regime.

Flow speed, flow rate, flow height, and avalanche volume at the stauchwall are determined in a similar way to that for the VS model using Equations (1) and (2). The differential equation for the flow speed in the track (steady-state flow regime with $Q=$ constant in a rectangular channel) has the following form

$$
u_{\mathrm{o}} u_{0}^{\prime}=g\left(\sin \Psi-\mu \cos \Psi-\frac{u_{0}^{3} B(x)}{\xi Q_{\mathrm{r}}}-\frac{2 u_{0}^{2}}{\xi B(x)}\right)
$$

with

$$
\tan \bar{\Psi}=\operatorname{mean}[\tan \Psi(x)]_{x}^{x-l(x)} ; \quad \text { and } l(x)=0.7 \xi d(x) / g .
$$

Local variations in flow height are assumed to be small, therefore terms including $\partial d / \partial x$ are neglected.

In the case of flow through dense forest, an effective friction coefficient $(\xi=\xi d(x)$, depending on number of trees per unit area and mean trunk thickness, is determined iteratively.

Sliding motion in the run-out is modelled in different ways. Mean flow height is expressed as

$$
\bar{d}_{0}=d_{\mathrm{p}}+\frac{u_{\mathrm{Op}}^{2}}{4 g \lambda_{\mathrm{v}}} .
$$

The flow height at the beginning of the run-out develops as follows

$$
d(t)=d_{\mathrm{p}}+\frac{\left(u_{\mathrm{op}}^{2}-u_{0}^{2}(t)\right)}{2 g \lambda_{\mathrm{v}}} .
$$

Depending on the assumptions stated below, run-out speed is given by

$$
\begin{aligned}
u_{0}\left(x_{\mathrm{s}}\right) u_{0}^{\prime} & =g\left[\sin \bar{\Psi}_{0}-\mu \cos \bar{\Psi}_{0}-\frac{u_{0}^{2}}{\xi d_{0}(x)}\right] \\
\tan \bar{\Psi}_{0} & =\operatorname{mean}[\tan \Psi(x)]_{x_{\mathrm{p}}}^{x_{\mathrm{s}}}
\end{aligned}
$$

$u_{0}\left(x_{\mathrm{s}}\right) u_{0}^{\prime}=(\mathrm{g} / \mathrm{m}) \int_{x_{\mathrm{s}}}^{x_{\mathrm{s}}-L}\left[\sin \Psi(x)-\mu \cos \Psi(x)-\frac{u_{0}^{2}}{\xi q_{\mathrm{q}}(x)}\right] \frac{\partial m}{\partial x} \mathrm{~d} x$.

Case A. Calculation for a separated front element at $x_{\mathrm{S}}$ moving at a mean slope angle measured from the front to 
point $\mathrm{P}$, and having a mean flow height (Equation (9)) corrected only for variations of avalanche width.

Cases $B$ and $C$. Calculation for a separated element actually leaving point $\mathrm{P}$ on a mean slope measured from the actual front to point $P$, having either mean flow height (case $B$ Equation (9)) or actual flow height (case C, Equation (10)) and only corrected for variations of avalanche width. For cases $\mathrm{A}$ to $\mathrm{C}$, the avalanche body is considered as a flexible solid body and internal longitudinal stresses are ignored.

Cases $D$ and E. Modelling of the movement of an avalanche body of constant volume, $V$, and length, $L$, (case D) or of a growing length, $L=x_{\mathrm{S}}-x_{\mathrm{p}}$, (case E), taking longitudinal stresses within the flexible body into account. Each element leaving point $P$ keeps its flow height corrected only for variations of the avalanche width.

\section{PF model}

Calculations for this model basically describe a partly fluidized flow and are not based on a well-established theory, although they allow experimental investigation of the consequences of the different propositions made. Our current opinion is that full fluidization does not occur (Salm and Gubler, 1985), and we assume therefore a convex exponential flow-speed profile combined with a plug flow. This profile is mainly determined by the movement of coarse-grained snow, that is, of snow balls $10 \mathrm{~mm}$ in diameter. This coarse-grained material may be partially fluidized by its interaction with the macroscopic track roughness. The shape of the speed profile is defined by the fluidization height, $d_{\mathrm{F}}$, of the coarse-grained material, and by a typical fluctuation decay length, $\lambda$ (Gubler, 1987). This relative motion of snow balls occurs only if the fluidization pressure is high enough to support the flow above it. Energy is dissipated in partially inelastic collisions of the snow balls (Equation (20)). If partial fluidization is an important characteristic of the flow (Equation (24)), continuity is assumed, because this flow type may behave more like a fluid than a solid body. On the other hand, if the internal motion is negligible (plug flow), the flow is modelled as a laterally deformable flexible body with constant height. The fine-grained material consists of submillimetre ice-crystal fragments, and may act as an interstitial fluid. It is concentrated mainly in the bottom shear layer. The coarse-grained material moves on this bottom shear layer of fine-grained material. This thin bottom shear layer is energized by the shear itself and accounts for an additional retardation force (Equation (19)) depending on the gradient of the speed profile close to the avalanche bed interface. The degree of fluidization depends on fluidization pressure and granular temperature, which are proportional to the square of the mean fluctuation speed. The fluctuations and fluctuation speeds are assumed to be random in space. Dry friction is essential to finally stop the avalanche and is added in the conventional way, its contribution being only significant at low flow speeds. Three additional quite important mechanisms are also added: entrainment of loose snow from the track; decrease of the avalanche volume by loss of slower-moving snow near the bottom of the flow from the avalanche body depending on track surface roughness and the actual speed profile; and granulation of the snow in the release zone. The on-going granulation of entrained snow at the avalanche front is neglected. The flow chart (Fig. 1) and the main equations for the PF model are given below. The model is again quasi-one-dimensional, with allowance for variable flow height and a varying slope-perpendicular flow-speed profile.

Assumed slope-perpendicular flow-speed profile:

$$
\begin{array}{rlrl}
u(y) & =u_{0} N[1-\exp (-y / \lambda)] & & \text { for } y \leqslant d_{\mathrm{F}} \\
u(y) & =u_{0} & & \text { for } y>d_{\mathrm{F}} \\
\text { where } & N=\left[1-\exp \left(-d_{\mathrm{F}} / \lambda\right)\right]^{-1} . &
\end{array}
$$

Mean fluctuation speed (scalar) of fine-grained material:

$$
\begin{aligned}
& \qquad \bar{v}_{0}(x)=\left[u_{0}(x) N(1-\exp (-1)]^{n} C_{1} / \mu,\right. \\
& \text { where } \quad n=0.6, C_{1}=C_{1}(e)=0.14 \\
& \text { Height of fluidization: } \\
& \qquad d_{\mathrm{F}}(x)=\min \left[A_{\mathrm{R}}\left\{1-C_{2} \exp \left\{-u_{0}(x) /\left[g \lambda_{\mathrm{R}}^{2} /\left(2 A_{\mathrm{R}}\right)\right]^{\frac{1}{2}}\right\}\right\}+\right. \\
& \left.+\bar{w}_{0}^{-2} \lambda C_{3} /[2 g d(x) \cos \Psi(x)], d(x)\right], \\
& \text { where } \quad C_{2}=0 \text { or } 1 \text { (default), } \\
& C_{3}=1 .
\end{aligned}
$$

Mean fluctuation speed (scalar) of coarse-grained material:

$$
\bar{w}_{0}=u_{0}(x) A_{\mathrm{R}} 2 \pi / \lambda_{\mathrm{R}} N\left[1-\exp \left(-A_{\mathrm{R}} / \lambda_{\mathrm{R}}\right)\right] .
$$

Retardation forces:

$$
u_{0}(x) u_{0}^{\prime}(x)=g \sin \Psi(x)=\sum_{i} k_{i} .
$$

Dry friction:

$$
k_{1}=-g f_{1} \cos \Psi(x) \quad \text { where } f_{1}=f_{1}\left(u, v_{0}\right)=1 .
$$

Shear friction:

$$
k_{2}=-c_{S} l / r \bar{v}_{0}(x) u_{0}(x) f_{2} \min \left\{0.5 \bar{v}_{0}{ }^{2} /[d(x) g \cos \psi], l\right\}
$$

where $f_{2}=f_{2}[d(x)]=1$.

Coarse-grain collisions:

$$
\begin{gathered}
k_{3}=-\theta A_{\mathrm{R}}^{3}\left(2 \pi / \lambda_{\mathrm{R}}\right)^{3} N^{3}\left(1-\exp \left(-A_{\mathrm{R}} / \lambda\right)\right)^{3} \\
{\left[1-\exp \left(-3 d_{\mathrm{F}} / \lambda\right)\right] u_{0}^{4} /\left(d(x) \lambda \overline{u^{2}}\right)}
\end{gathered}
$$$$
\text { where } \overline{u^{2}}=(1 / d) \int_{0}^{d} u^{2}(y) d y
$$

Granulation:

$$
k_{4}=-0.125 \sigma^{*} C_{4} \exp \left(-\frac{1.5 \sigma^{*} C_{4}}{\rho_{\mathrm{S}} \theta_{\mathrm{F}} \bar{w}_{0}^{2}}\right) \frac{2 \pi}{\lambda \rho \theta_{\mathrm{F}}} N\left(1-\exp \left(-\frac{A_{\mathrm{R}}}{\lambda}\right)\right] \frac{u_{0}^{2}}{\overline{u_{0}^{2}}},
$$

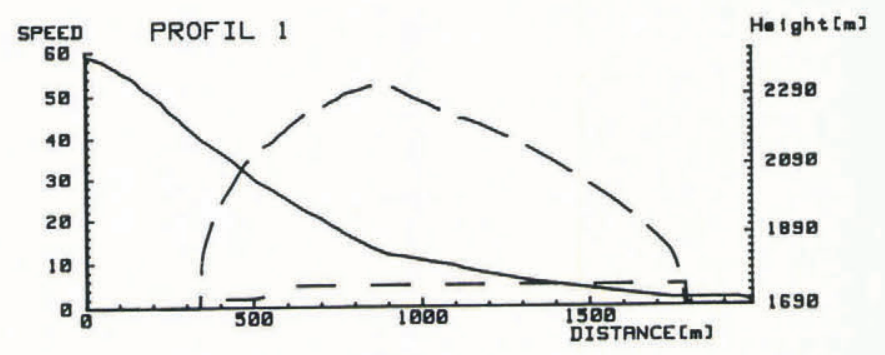

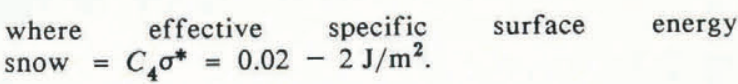

for

Fig. 1. Flow chart for PF model.

Entrainment:

$$
k_{5}=-0.5 \rho_{\mathrm{S}} B(x) d_{\mathrm{E}}(x) u_{0}(x) /(\rho V) .
$$

Fluctuation energy of the coarse-grain material available for granulation, if mean grain diameter $>1 \mathrm{~mm}$, and for dissipation in non-elastic collisions between snow balls is:

$$
T(x)=\bar{w}_{0}^{2}(x) \lambda\left[1-\exp \left(-2 d_{\mathrm{F}} / \lambda\right)\right] /\left(2 d_{\mathrm{F}}\right) .
$$


Conditions for partially fluidized flow $(Q=$ constant $)$ and sheet flow $(d=$ constant $)$ are:

$$
\begin{array}{lll}
0.5 w_{0}^{2}(x)<C_{5} g d \cos \Psi & \text { sheet flow, } & \\
0.5 w_{0}^{2}(x) \geqslant C_{5} g d \cos \Psi & \text { fluidized flow, } & C_{5}=0.7
\end{array}
$$

Variations of the avalanche volume by entrainment and loss of snow are given by:

$$
\begin{gathered}
\mathrm{d} V / \mathrm{d} x=B(x)\left[d_{\mathrm{V}}\left\{N-\lambda / d_{\mathrm{F}}\right) d_{\mathrm{F}} / d+\left(d-d_{\mathrm{F}}\right) / d-N\right. \\
\left.\left.\left[1-\lambda / d_{\mathrm{V}}\left[1-\exp \left(-d_{\mathrm{V}} / \lambda\right)\right]\right\}\right\}+d_{\mathrm{E}}\right] .
\end{gathered}
$$

$B\{\}=$ snow loss; $B d_{\mathbf{E}}=$ snow entrainment;

$$
d_{\mathrm{v}}=\min \left[d_{\mathrm{F}}, \max \left(\lambda, A_{\mathrm{R}}\right)\right] .
$$

If sliding of a compact sheet is modelled; for $\tan \Psi<\mu$, $d=$ constant, $k_{3}=k_{4}=k_{5}=0$, and

$$
k_{6}=(g / L) \int^{\max \left(x-L, x_{\mathrm{p}}\right)} \mathrm{d} x^{\prime}\left[\sin \Psi\left(x^{\prime}\right)-\mu \cos \Psi\left(x^{\prime}\right)\right] / \cos \Psi .
$$

Centripetal stresses are neglected in these equations.

\section{CHARACTERISTIC FEATURES OF THE MODELS}

If instructions for determination of longitudinal profile segmentation and the position of point $P$ using the rules mentioned earlier are followed exactly, the VS model parameter set $\mu=0.155, \xi=1000$ produces reliable run-out predictions for large but not extensively channelled dry-snow avalanches, except that resulting maximum flow speeds are about $30 \%$ below measured speeds. Although it is possible to find, for a set of very similar avalanches, a parameter pair that would fit maximum speed and run-out, this same pair would never correctly model avalanches of different track characteristics. Because of the speed-squared dependence (speed cubed for $Q=$ constant) of the retardation force, it is an inherent property of the Voellmy models that they result in too low maximum speeds at least for large avalanches. For small avalanches $(\mu=0.2-0.3)$ the differences between modelled and measured speeds are smaller and less significant.

This basic drawback applies also to the VSG model. Typical results for the different assumptions made for run-out calculations (see description of VSG model) are shown for an avalanche with a very long run-out and significantly varying slope angle (Ariefa, Samedan, Engadin) for which the release zone is not particularly large, the slab volume amounts to about $90000 \mathrm{~m}^{3}$ with a release height of $1.0-1.3 \mathrm{~m}$, the slab width is $160 \mathrm{~m}$, and the mean slope angle is $39^{\circ}$. The avalanche is slightly channelled to a width of $60 \mathrm{~m}$, the mean slope angle of the track is $27^{\circ}$, and of the run-out segment $8^{\circ}\left(\tan ^{-1} \mu=8.8^{\circ}\right)$. Within this $900 \mathrm{~m}$ run-out segment there is a steeper $200 \mathrm{~m}$ segment in the real longitudinal profile with slope angles slightly greater than $9^{\circ}$. The measured run-out distance is between 860 and $900 \mathrm{~m}$. With the parameters mentioned above, the VS model predicts a run-out distance of $875 \mathrm{~m}$, but only if, in spite of the existence of the steeper part, one uses the one-segment approximation for the run-out. The corresponding results for the VSG models are summarized in Table I.

If the VSG calculations are made with respect to an idealized segmented longitudinal profile with slope angles corresponding to those used with the VS model, VSG A and $B$ produce almost identical results (nos. 2, 3 in Table I). If the avalanche length is limited by the avalanche volume, then the run-out distance is significantly reduced, because of the resulting lower mean flow height of the sliding avalanche body (no. 5 in Table I). A reduced run-out distance follows also from a VSG A calculation using the actual slope angle at the avalanche front instead of the actual mean angle taken between point $P$ and the front (Table I, no. 7).

Modelling along the real longitudinal profile (Fig. 2) allows for a switch back from sliding $(Q \neq$ constant $)$ to flowing $(Q=$ constant $)$ on the steeper section, and also drastically reduces the modelled run-out distance. The reason for this is a reduced flow height (reduced $u_{o p}$ ) in the second run-out segment compared with the first one. If this switch back from sliding to flowing is inhibited, an increased actual mean slope angle for the lower section of the run-out (Equation (11)) compared with the mean run-out segment slope angle pushes the avalanche further into the flat region (Table $\mathrm{I}$, nos. 11, 12). If the avalanche length is again limited by the avalanche volume, the modelled run-out distance corresponds well to the observed one (Table I, no. 13). Very similar experiences have been gained with respect to a large variety of relatively steep run-out profiles. This last type of run-out modelling is the most logical, because it limits slope-angle averaging to the true length of the sliding body.

From this comparison of different modelling assumptions it can be learnt that choice of the point $\mathrm{P}$, and decisions on how further to segment the run-out, are very

\begin{tabular}{|c|c|c|c|c|c|}
\hline 1 & VS & & segment & 875 & 9.8 \\
\hline 2,3 & VSG & A, B & segment & 875 & $9.3,9.8$ \\
\hline 4 & VSG & C & segment & 855 & $5.0-16.5$ \\
\hline 5 & VSG & D & segment & 580 & $5.0-10.0$ \\
\hline 6 & VSG & E & segment & 915 & $5.0-16.5$ \\
\hline 7 & VSG & A & $\begin{array}{l}\text { real, no } \\
\text { averaging }\end{array}$ & 630 & 9.8 \\
\hline 8 & VSG & A & $\begin{array}{l}\text { real, with } \\
\text { averaging }\end{array}$ & 690 & 6.4 \\
\hline 9 & VSG & $\mathrm{C}$ & averaging & 660 & $4.5-8.5$ \\
\hline 10 & VSG & D & averaging & 710 & $4.5-9.0$ \\
\hline 11 & VSG & A & averaging & 980 & 9.8 \\
\hline 12 & VSG & $\mathrm{C}$ & averaging & 980 & $5.0-18.0$ \\
\hline 13 & VSG & D & averaging & 860 & $2.0-10.0$ \\
\hline
\end{tabular}
critical. Using the VSG D model produces the flow-rate dependence and also an avalanche-volume dependence for the run-out distance of which tends to reduce very long

TABLE I. RUN-OUT MODELLING WITH VSG MODEL USING DIFFERING ASSUMPTIONS

No. Model Profile Run-out $\begin{gathered}\text { Deposition } \\ \text { height }\end{gathered}$ Remarks

(m) (m) one segment approx. for run-out equivalent to VS model

reduced speed in first part of run-out deposit length $230 \mathrm{~m}$

similar to no. 4

calculation refers to actual slope angle at front

run-out interrupted by $200 \mathrm{~m}$ track segment

run-out interrupted by $200 \mathrm{~m}$ track segment

run-out interrupted by $200 \mathrm{~m}$ track segment

switch back to $Q=$ constant flow

within run-out inhibited

similar to no. 4

deposit length $210 \mathrm{~m}$ 


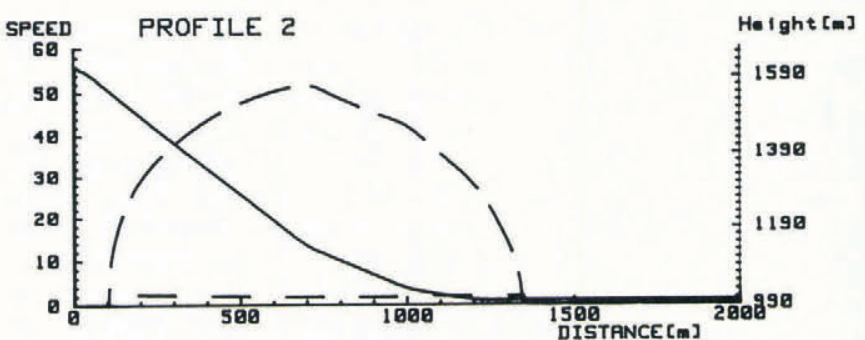

Fig. 2. Longitudinal profile of Ariefa avalanche (profile 1) and avalanche simulation using standard PF model parameters. Upper broken curve is flow speed $(\mathrm{m} / \mathrm{s})$, lower broken curve is flow height (left scale $0-30 \mathrm{~m}$ ).

run-out for low-volume avalanches but has no effect for very large avalanches on steep long run-out or smaller avalanches on low-angle short run-out. If an avalanche flows through a dense forest, additional friction retards the flow. Theoretically, this friction will be proportional to the square of the flow speed, to the cross-sectional area of the trees within the flow, and to a friction coefficient. Modelling is then carried out by replacing $\xi$ by an effective $\xi(u, d, Q$, forest-characterization, friction coefficient). Assuming continuity, flow height will increase but speed decrease and the resulting snow pressures will eventually damage the forest. Unfortunately, there is only a low degree of certainty in the value to be assigned to the friction parameter (Frey and others, 1987). Preliminary calculations show that for a dense forest (mean tree distance $5.3 \mathrm{~m}$ apart, tree diameter $0.27 \mathrm{~m}$ ) the effective $\xi$ value will be reduced to $100-200 \mathrm{~m} / \mathrm{s}^{2}$.

The main difference between the Voellmy-Salm-type models and the PF model is the number of velocitydependent retardation forces and the variation of their relative importance as a function of bed roughness, flow depth, and flow speed. The basic model parameters, $c_{\mathrm{S}}$, (basal shear friction); $\mu$ (dry-friction coefficient); $\lambda$ (decay length of coarse-grain fluctuation); and the power, $n$, in the determination of the shear fluctuation

$$
\left.\mathrm{v}_{0} \propto\left(\frac{\mathrm{d} u}{\mathrm{~d} y}\right)^{n}\right|_{y=0}
$$

have been calibrated using measured speed and run-out data and are kept constant in all calculations. The PF model is by far the most sensitive to small changes of topographical, bed-roughness and avalanche-size parameters of the models; the flow regime flip between partial fluidized flow and sliding depending on coarse-grain fluctuation pressure, speed, and flow height. Fixed combinations of roughness parameters are used to characterize typical track types (Table II). The material-dependent parameters $c_{\mathrm{S}}=0.008$, $\mu=0.2, \lambda=0.3 \mathrm{~m}, \quad n=0.6$ are kept constant in all calculations for dry-snow avalanches.

Some of the main features of the PF model will be discussed for two extreme avalanche paths Ariefa (profile 1, Fig. 2), which is slightly confined as described above, and an unconfined synthetic path (profile 2, Fig. 3). The different contributions to the acceleration of the avalanche body at the distances 300 and $900 \mathrm{~m}$ of profile 2 of the roughness $R_{\mathrm{R}}=A_{\mathrm{R}} / \lambda_{\mathrm{R}}$ and the roughness amplitude $A_{\mathrm{R}}$ are shown in Figure $4 a$ and $b$. In the initial steep part of the

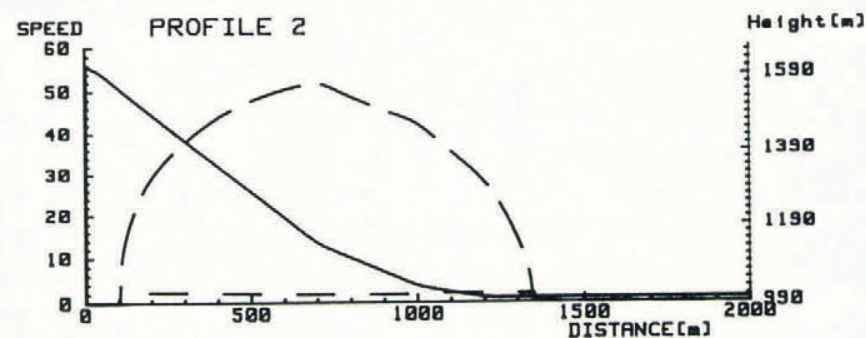

Fig. 3. Longitudinal profile of synthetic path (profile 2) and simulation of unconfined avalanche using standard PF model parameters.

track, a distance $\mathrm{d} t$ of $300 \mathrm{~m}$, the acceleration of the avalanche body, increases strongly with decreasing roughness for low to intermediate roughness. For an unconfined avalanche on smooth terrain, where $R_{\mathrm{R}}=0.02$, collisional dissipation is much smaller than shear dissipation. For a similar flow on rough terrain, or in a channel, retardation by collisional dissipation is higher than by basal shear. At $900 \mathrm{~m}$, retardation increases moderately with decreasing roughness and roughness amplitude. For low roughness, retardation increases more rapidly due to the strong increase of shear dissipation with decreasing roughness. This is explained by a fast-decreasing fluidization height causing a sharp increase of the basal shear-velocity gradient and shear-fluctuation speed (Equation (14)). Retardation induced by coarse-grain fluidization increases significantly with increasing roughness, and is the most important dissipative process in well-fluidized high-speed flow on rough terrain. From Figure 5, it can be seen that avalanche speed at a given point decreases rapidly with increasing roughness due to increasing collisional dissipation. At the same time, flow height increases but avalanche flow rate and volume decrease because of snow loss from the avalanche body.

The variations of the different contributions to the acceleration of the avalanche body along the path again indicate the lower significance of collisional dissipation for an unconfined avalanche compared with a confined avalanche (Fig. 6). In the run-out, at low speeds, retardation is mainly done to shear dissipation and dry friction. These properties of the model clearly explain the large variability of the run-out distance as a function of track roughness (Fig. 5). In Figure 7 a similar plot is shown for the Ariefa avalanche. Collisional dissipation always remains low, the avalanche is usually sliding and there is no partial fluidization. If the avalanche flow rate or flow height is reduced below a certain limit by reducing release height below $1.1 \mathrm{~m}$, the low flow height or low internal pressure would allow for partial fluidization, causing significantly increased retardation because of the assumption of constant flow rate for PF flow instead of constant flow height for sliding motion. The following rules can be deduced from PF modelling:

Flow speed and run-out distance increase with avalanche volume.

Increasing roughness decreases avalanche volume, speed, and run-out.

Small avalanches may stop on steep terrain if flow height becomes very small.

\section{TABLE II. STANDARDIZED ROUGHNESS PARAMETERS FOR PF MODEL}

Track type

Release zone, open slope

Unconfined track, open slope

Confined track, channel

Cliffs, rough slope, forest

Piles to break an avalanche,

extremely rough, houses

Open run-out
Amplitude

$A_{R}$

(m)

0.5

$0.2-0.3$

$0.3-0.5$

$0.4-0.5$

1.0

$0.2-0.3$
Wavelength

$R_{\mathrm{R}}=A_{\mathrm{R}} / \lambda_{\mathrm{R}}$

\section{$\lambda_{R}$}

(m)

20

10

10

10

10-20

10
0.025

$0.02-0.03$

$0.03-0.05$

$0.04-0.05$ 
ACCELERATION, COLLISION, $300 \mathrm{~m}$

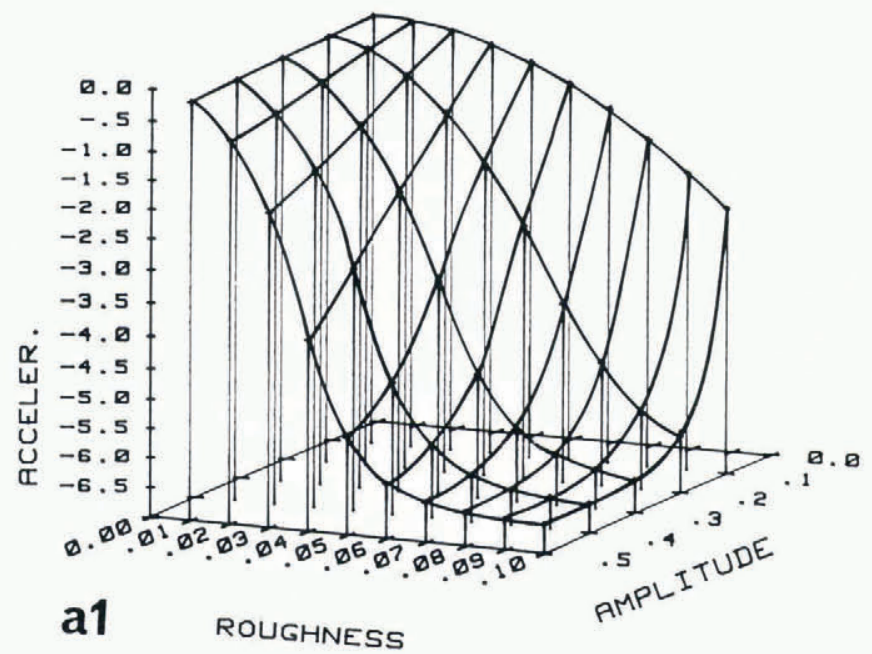

-ACCELERATION, SHERR, 300m

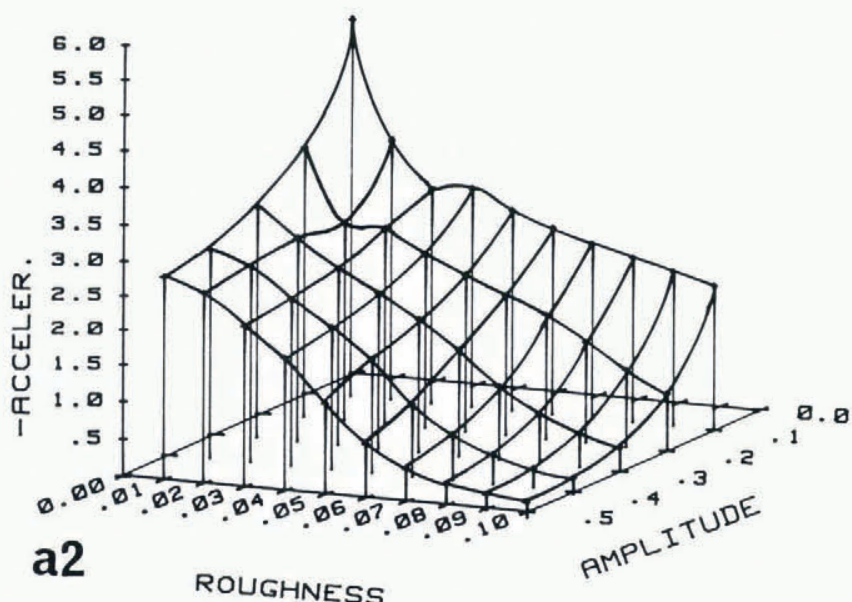

ACCELERATION, TOTAL, 300m

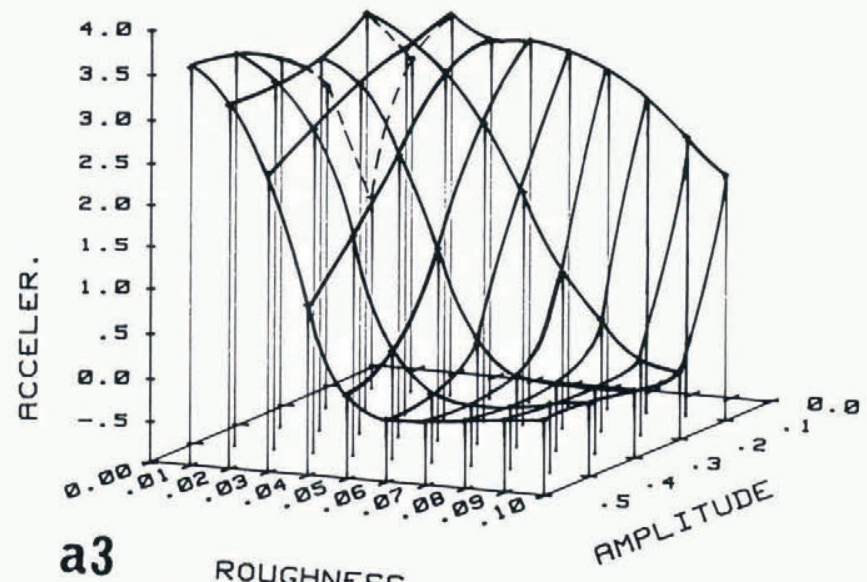

PCCELERATION, COLLISION, $900 \mathrm{~m}$

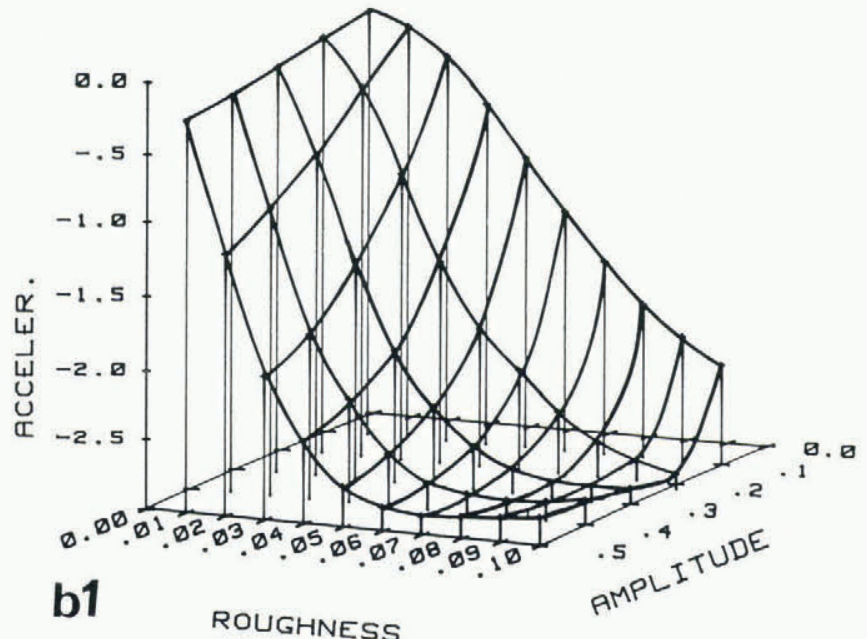

-ACCELERATION, SHEAR, 900m

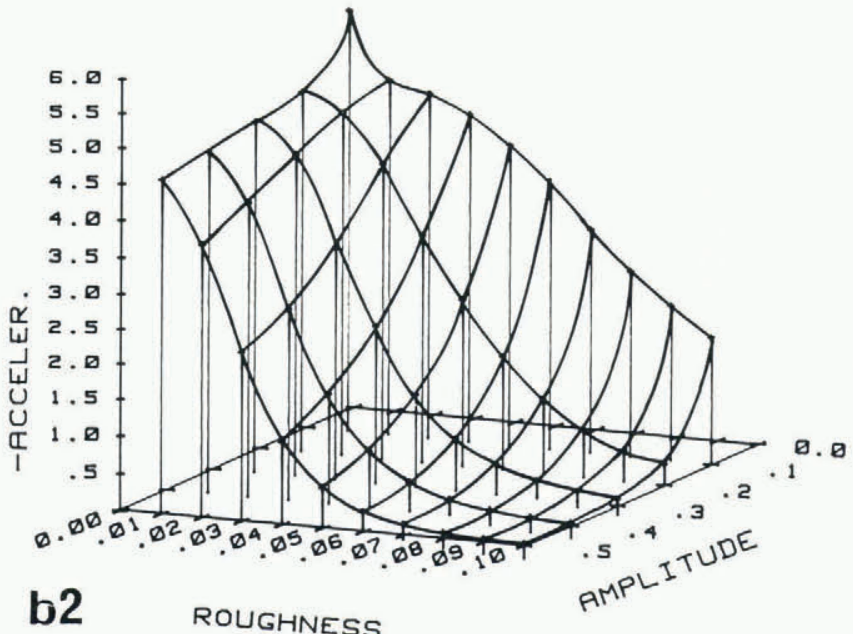

-ACCELERATION, TOTAL, 900m

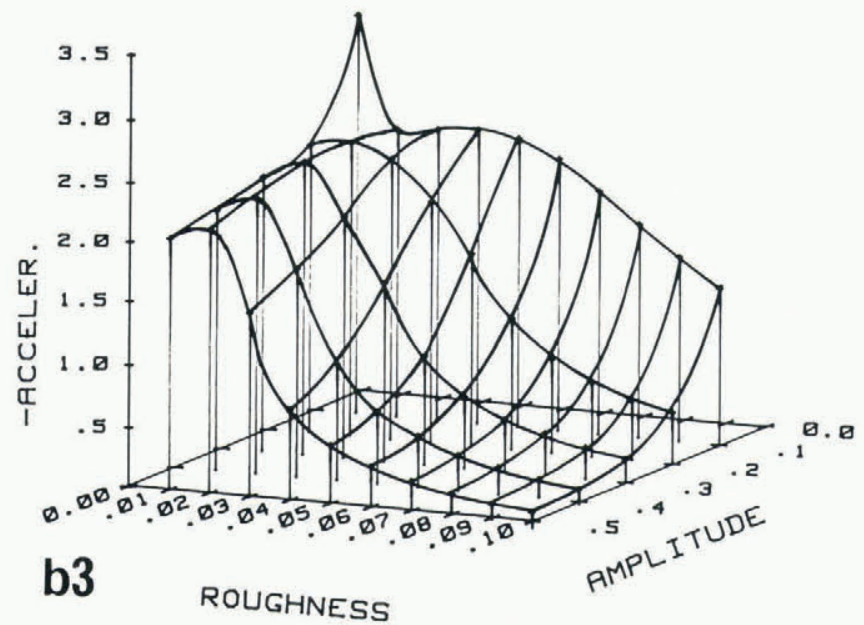

Fig. 4. Contributions to total acceleration of avalanche body by shear dissipation, collisional dissipation, and resulting total acceleration at $300 \mathrm{~m}$ (a) and $900 \mathrm{~m} \mathrm{(b)}$ as function of roughness on profile 2 (acceleration in $\mathrm{m} / \mathrm{s}^{2}$; amplitude in $\mathrm{m}$ ). 
AVRL, SPEED (ROUGHNESS), 600m

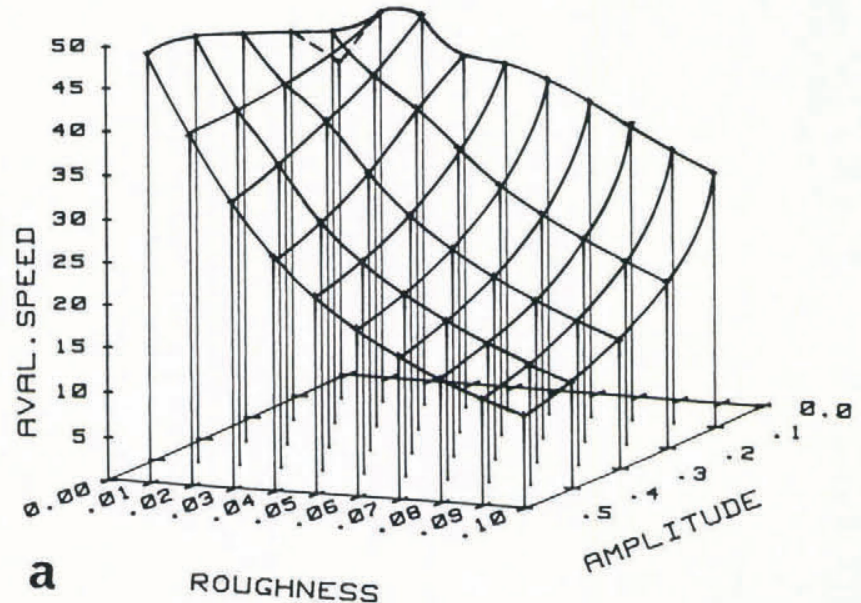

RVAL.VOLUME (ROUGHNESS), 600m

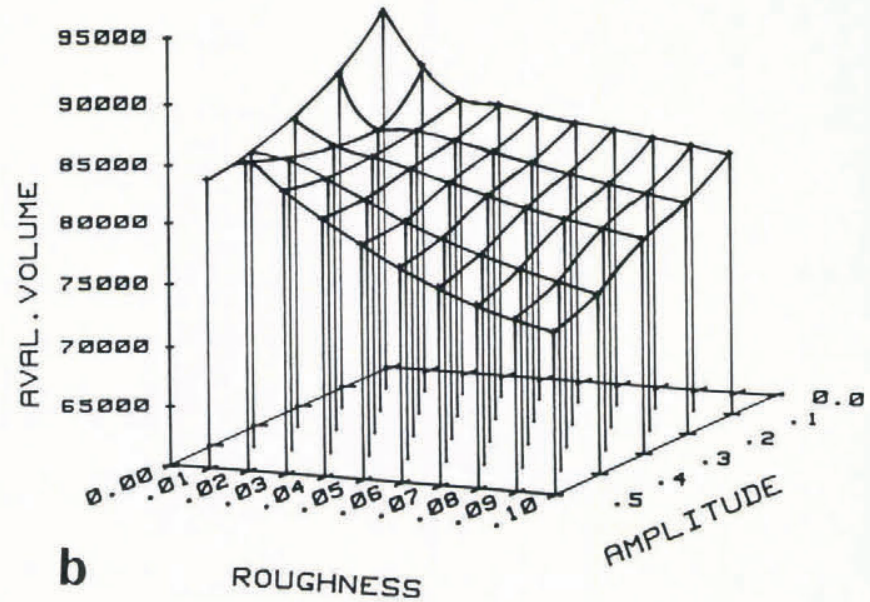

RUN-OUT (ROUGHNESS)

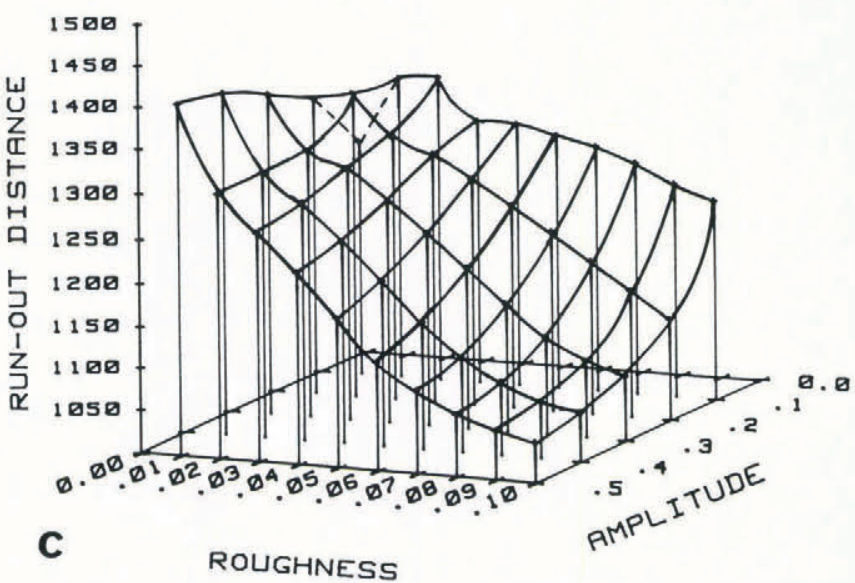

Fig. 5. Avalanche speed $(\mathrm{m} / \mathrm{s})$, volume $\left(\mathrm{m}^{3}\right)$ at $600 \mathrm{~m}$, and run-out distance $(\mathrm{m})$ for unconfined avalanche in profile 2 as function of roughness.
COLLISION (1), SHEAR (2), GRAVITY-DRY FRICT. (3)

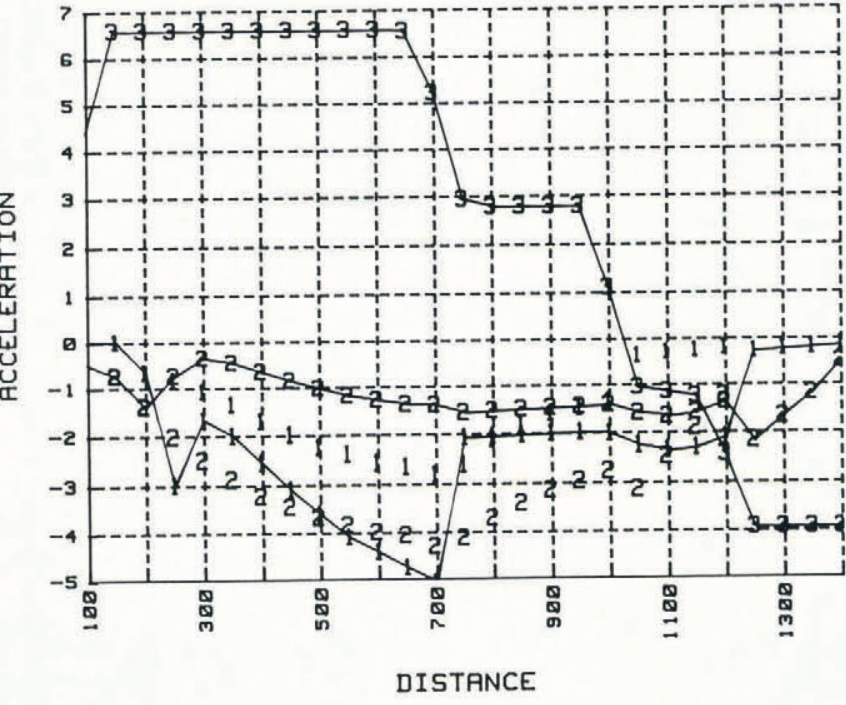

Fig. 6. Longitudinal profile of different contributions to total acceleration $\left(\mathrm{m} / \mathrm{s}^{2}\right)$ for an unconfined avalanche (points not connected) and a confined avalanche (points connected) in profile 2 .

COLLISION (1), SHEAR (2), GRAVITY-DRY FRICT. (3)

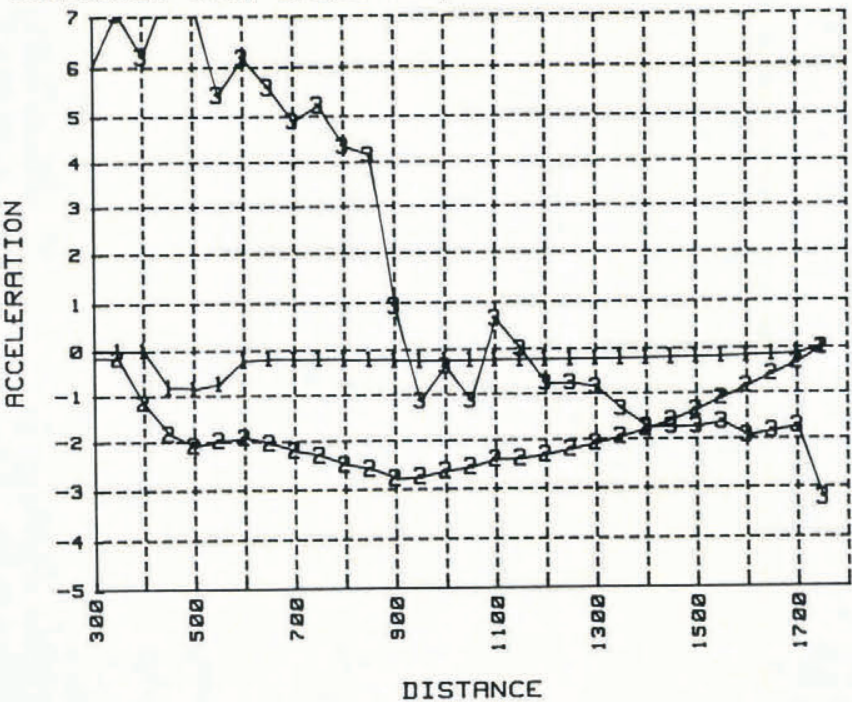

Fig. 7. Longitudinal profile of different contributions to total accleration $\left(\mathrm{m} / \mathrm{s}^{2}\right)$ for Ariefa path (profile 1).

If partial fluidization occurs, flow height increases with increasing roughness.

Sliding avalanches have longer run-out than partial fluidized avalanches.

High flow height and low roughness avoid fluidization and result in longer run-out.

High roughness amplitudes at beginning of run-out significantly reduce run-out distance.

These results are in good correspondence with observations and experience (Gubler, 1987).

\section{COMPARISON OF THE THREE MODELS}

Calculations have been based on data for 25 large avalanches. Most of the field data have been collected by Frutiger and are carefully described in SAR reports. The avalanches can be subdivided into four categories: (1) avalanches with horizontal run-out, (2) avalanches that reach a counterslope, (3) steep, long run-out, and (4) run-out with significantly varying slope angles. These avalanches have the following specifications: volume $=14-200 \times 10^{3} \mathrm{~m}^{3}$; flow rate $=1.7-21 \times 10^{3} \mathrm{~m}^{3} / \mathrm{s}$, track length $=800-5500 \mathrm{~m}$; fall heights from stauchwall to avalanche tip $=230-1500 \mathrm{~m}$; 
mean elevation angle from avalanche tip to stauchwall $=11-25^{\circ}$. Initial avalanche volumes are estimated from fracture-line observations, topography, and meteorological data. Avalanche width is evaluated from observations and topographical restrictions. Entrainment (loose snow in the track) and roughness parameters (modifying drag and snow loss) are selected according to the fixed rules mentioned above.

The results obtained are as follows:

Maximal speed: the PF calculations result in significantly higher speeds than do the Voellmy models, the ratio amounts to $1: 1.6 \pm 0.3$ (range $1: 1.1-2.16$ ). The PF model has been calibrated using measured speeds. The modelled speeds differ more for large avalanches than for smaller ones.

Run-out distance: in only very few cases release area and mean slab thickness have been measured exactly, so these parameters together with avalanche width can be varied within the limits given by observation, experience, and topography to match observed run-out distance. In almost all cases it was possible to match the observed run-out distance within a few tens of metres; the least-critical models are the VSG D and the PF model, the latter being more critical with respect to flow height because of possible flow-regime changes. Nevertheless, some trends can be summarized. For category (1) avalanches, Voellmy-Salm-type models tend to predict run-out distances too small if the horizontal run-out segment is modelled with a slope angle of $0^{\circ}$; because of the higher speeds at the beginning of the run-out, the PF models give correct results. Prediction of category (3) and (4) avalanches is very critical because calculated run-out distances often depend strongly on the segmentation; for these avalanche paths, it is especially important to be able to run models with respect to digitized longitudinal profiles.

Height of deposition near avalanche tip: the VS model of ten results in estimates for the mean deposition heights which seem very large compared with observed values. The calculated PF heights are lower by a factor of $2.7 \pm 0.9$ with a range of 1-4.95. The VSG D model enables the operator to calculate longitudinal deposition height profiles; the PF model determines either the sliding speed and position of an independent front element of the avalanche or calculates speed and position of the whole avalanche body reaching the run-out modelled as a flexible solid body. An increase in flow height of the rearward flow that runs against the sliding slab is not taken into account.
These are two extreme assumptions, reality being somewhere in between. Often pushing of frontal parts of an avalanche by backward segments can be observed; modelling of such a mechanism is very difficult.

\section{CONCLUDING REMARKS}

Although the PF model is unsuitable for operational use by consultants, it is the only model that correctly models flow speed and run-out; it can therefore be used to recalibrate and investigate limitations of simpler models, such as the VS model, which are now widely used in consulting work. To further improve modelling, we must refine calculations and, even more importantly, measure and observe more carefully, so as to get many more complete data sets for different avalanche-path and snow conditions, including release zone, speed, entrainment, snow loss and deposition measurements. Avalanche researchers already know what a difficult and of ten demotivating task this is.

\section{REFERENCES}

Buser, O. and H. Frutiger. 1980. Observed maximum run-out distance of snow avalanches and the determination of the friction coefficients $\mu$ and $\xi$. J. Glaciol., 26(94), 121-130.

Frey, W., H. Frutiger, and W. Good. 1987. Openings in the forest caused by forest perishment and their influence on avalanche danger. Human impacts and management of mountain forests. Ibaraki, Japan, Forestry and Forest Product Research Institute, 223-238.

Gubler, H. 1987. Measurements and modelling of snow avalanche speeds. International Association of Hydrological Sciences Publication 162 (Symposium at Davos 1986 Avalanche Formation. Movement and Effects), 405-420.

Salm, B. Unpublished. Fliessübergänge und Auslaufstrecken von Lawinen. Weissfluhjoch/Davos, Eidgenössische Institut für Schnee- und Lawinenforschung. Interner Bericht.

Salm, B. and A. Burkard. Unpublished. Berechnung von Fliesslawinen. Weissfluhjoch/Davos, Eidgenössische Institut für Schnee- und Lawinenforschung.

Salm, B. and H. Gubler. 1985. Measurement and analysis of the motion of dense flow avalanches. Ann. Glaciol., 6 , 26-34.

Schaerer, P.A. 1975. Friction coefficient and speed of flowing avalanches. International Association of Hydrological Sciences Publication 114 (Symposium at Grindelwald 1974 - Snow Mechanics), 425-432. 